\title{
Aurora A and cancer
}

\author{
Stephanie Le Bras, Arun Prasath Damodaran and Claude Prigent* \\ Rennes University, CNRS, IGDR Institute of Genetics and Development of Rennes, France
}

Received: February 21, 2018; Published: April 06, 2018

*Corresponding author: Rennes University, CNRS, IGDR (Institute of Genetics and Development of Rennes), UMR 6290, Team labelled Cancer League 2014-2016, F-35000 Rennes, France, Tel: +33(0)223234731; Email: claude.prigent@univ-rennes1.fr

\section{Abstract}

Aurora-A is a serine-threonine kinase found over expressed in cancers and demonstrated to be an oncogene. Since the kinase regulates cell cycle progression several pharmaceutical companies were involved in the identification of inhibitors to be used in cancer treatments. Many of such inhibitors are now commercially available but none of them succeeded any phase III clinical trials, yet. The concern here is, whether Aurora-A is still a good target or biomarker?

\section{Introduction}

Aurora-A was discovered in 1995 as a protein kinase involved in centrosome separation in Drosophila [1]. In 1997 a human cDNA related to Aurora-A and named BTAK (Breast Tumour Activated Kinase) was identified to be over expressed in breast cancers [2]. One year later the kinase was demonstrated to behave like an oncogene whose over expression was sufficient to induce tumour formation $[3,4]$. The first function associated with the kinase was bipolar spindle assembly and the first localisation was centrosomal [5]. So the idea was that over expression of Aurora-A would disturb bipolar spindle assembly leading to mitotic defects at the origin of genome instability that would, in turn, lead to cancer. This was indeed nicely demonstrated in mice where over expression of Aurora-A in mammary epithelia is sufficient to induce tumour formation but only after a prolonged period of genomic instability [6]. This kind of tumours derived from Aurora-A over expression in mice even showed the same gains and losses of genes expression like the ones observed in human breast cancers [7].

The prolonged genomic instability necessary for cells over expressing Aurora-A to form tumours, clearly suggests that an accumulation of genetic events is responsible for the origin of cancer. This has been also observed in mouse through an accumulation of gene mutations [7]. Having said that, is Aurora-A a good target for cancer treatment? The number of failures in various phase III clinical trials clearly indicate that inhibiting Aurora-A is not sufficient. However, Aurora-A is a good biomarker. The simple fact that tumours have conserved the over expression of the kinase, strongly indicate that it should be advantageous to them. And indeed, high level of Aurora-A has been demonstrated to help cancer cells to become resistant to chemotherapeutic drugs. Etoposide or cisplatin, for instance, are is inducing DNA damages that are detected by a cell cycle checkpoint which in turn arrests cell cycle progression either to repair the damages or to induce cell death. Cells over expressing Aurora-A have been shown to override this checkpoint as they keep dividing even in the presence of DNA damages [8-11]. The same is true for taxanes that are microtubule poisons used in chemotherapies. Taxanes induce cell cycle arrest in mitosis due to the fact that the spindle assembly checkpoint remains active, and this arrest eventually leads to cell death. Again cells over expressing Aurora-A override the mitotic checkpoint and keep dividing [12,13].

Detecting the over expression of Aurora-A in cancer cells can then be useful to adapt treatments like supplementing or complementing Aurora-A inhibitors to DNA damaging agents or to microtubule poisons in the cancer cells over expressing Aurora-A to counteract chemo resistances [14-16]. For these reasons even if many phase III clinical trials failed, the search for Aurora-A inhibitors should be pursued. The last comment to be done on Aurora-A and cancer is that basic research on Aurora-A should also be reinforced since numerous non-canonical functions of the kinase have been discovered in these past few years that have a crucial impact on cancer research [17]. One does not completely understand yet, how Aurora-A triggers cancers.

\section{References}

1. Glover DM, Leibowitz MH, McLean DA, Parry H (1995) Mutations in aurora prevent centrosome separation leading to the formation of monopolar spindles. Cell 81(1): 95-105.

2. Sen S, Zhou H, White RA (1997) A putative serine/threonine kinase encoding gene BTAK on chromosome 20q13 is amplified and overexpressed in human breast cancer cell lines. Oncogene 14(18): 2195-2200. 
3. Bischoff JR, Anderson L, Zhu Y, Mossie K, Ng L, et al. (1998) A homologue of Drosophila aurora kinase is oncogenic and amplified in human colorectal cancers. EMBO J 17(11): 3052-3065.

4. Zhou H, Kuang J, Zhong L, Kuo WL, Gray JW, et al. (1998) Tumour amplified kinase STK15/BTAK induces centrosome amplification, aneuploidy and transformation. Nat Genet 20(2): 189-193.

5. Roghi C, Giet R, Uzbekov R, Morin N, Chartrain I, et al. (1998) The Xenopus protein kinase pEg2 associates with the centrosome in a cell cycledependent manner, bind to the spindle microtubules and is involved in bipolar mitotic spindle assembly. J Cell Sci 111(Pt 5): 557-572.

6. Wang X, Zhou YX, Qiao W, Tominaga Y, Ouchi M, et al. (2006) Over expression of aurora kinase $A$ in mouse mammary epithelium induces genetic instability preceding mammary tumor formation. Oncogene 25(54): 7148-7158.

7. Treekitkarnmongkol W, Katayama H, Kai K, Sasai K, Jones JC, et al. (2016) Aurora kinase-A overexpression in mouse mammary epithelium induces mammary adenocarcinomas harboring genetic alterations shared with human breast cancer. Carcinogenesis 37(12): 1180-1189.

8. Yang H, He L, Kruk P, Nicosia SV, Cheng JQ (2006) Aurora-A induces cell survival and chemoresistance by activation of Akt through a p53dependent manner in ovarian cancer cells. Int J Cancer 119(10): 23042312.

9. El-Sheikh A, Fan R, Birks D, Donson A, Foreman NK, et al. (2010) Inhibition of Aurora Kinase A enhances chemosensitivity of medulloblastoma cell lines. Pediatr Blood Cancer 55(1): 35-41.

10. Krystyniak A, Garcia-Echeverria C, Prigent C, Ferrari S (2006) Inhibition of Aurora A in response to DNA damage. Oncogene 25(3): 338-348.
11. Cazales M, Schmitt E, Montembault E, Dozier C, Prigent C, et al. (2005) CDC25B phosphorylation by Aurora-A occurs at the G2/M transition and is inhibited by DNA damage. Cell Cycle 4(9): 1233-1238.

12. Jiang Y, Zhang Y, Lees E, Seghezzi W (2003) Aurora A overexpression overrides the mitotic spindle checkpoint triggered by nocodazole, a microtubule destabilizer. Oncogene 22(51): 8293-8301.

13. Anand S, Penrhyn-Lowe S, Venkitaraman AR (2003) AURORA-A amplification overrides the mitotic spindle assembly checkpoint, inducing resistance to Taxol. Cancer Cell 3(1): 51-62.

14. Chiba Y, Sato S, Itamochi H, Yoshino N, Fukagawa D, et al. (2017) Inhibition of Aurora Kinase A Synergistically Enhances Cytotoxicity in Ovarian Clear Cell Carcinoma Cell Lines Induced by Cisplatin: A Potential Treatment Strategy. Int J Gynecol Cancer 27(8): 1666-1674.

15. Huck JJ, Zhang M, Mettetal J, Chakravarty A, Venkatakrishnan K, Zhou X et al. (2014) Translational exposure-efficacy modeling to optimize the dose and schedule of taxanes combined with the investigational Aurora A kinase inhibitor MLN8237 (alisertib). Mol Cancer Ther 13(9): 21702183.

16. Li Y, Tang K, Zhang H, Zhang Y, Zhou W, et al. (2011) Function of Aurora kinase A in Taxol-resistant breast cancer and its correlation with P-gp. Mol Med Rep 4(4): 739-746.

17. Pérez de Castro I, Carmena M, Prigent C, Glover DM (2017) Editorial: Aurora Kinases: Classical Mitotic Roles, Non-Canonical Functions and Translational Views. Front Oncol 7: 48.

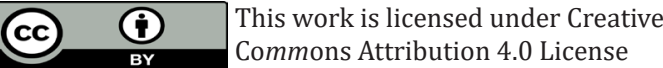

Submission Link: https://biomedres.us/submit-manuscript.php

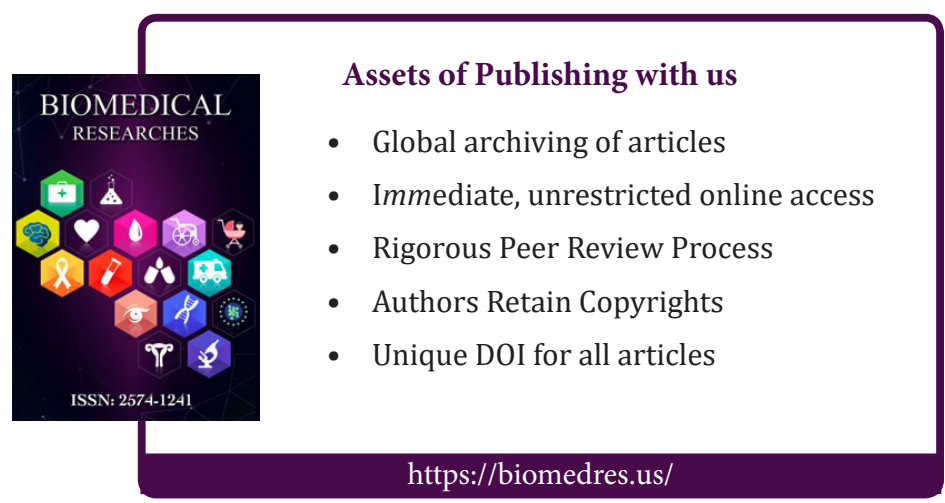

